\title{
Active Design of Hard Disk Suspension Assemblies Using Multiobjective Optimization Techniques
}

\author{
Yee-Pien Yang and Chin-Chung Kuo \\ Department of Mechanical Engineering \\ National Taiwan University \\ Taipei, Taiwan 106, R.O.C. \\ 02-363-0231 Ext.2175 ypyang@w3.me.ntu.edu.tw
}

\begin{abstract}
The active designs of hard disk suspension assemblies is formulated as multiobjective optimization problems. This design methodology integrates the structure and control objectives, which consist of natural frequencies and an optimal control performance index with weighted system state regulation errors and control efforts. Preloading and air bearing effects are both considered in the optimal design. The interface program communicates between the finite element analyzer and the optimizer with two multiobjective optimization techniques goal programming and compromise programming. The feasibility of the optimal design is demonstrated and the frequency responses are investigated.
\end{abstract}

\section{Introduction}

This paper presents a multiobjective optimization design on the suspension assemblies. The integrated structure and control optimization is applied to the actively controlled suspension assemblies, where the performance index of the linear quadratic regulator is incorporated with frequency objectives.

\section{Modeling of Suspension Assembly}

The hard disk suspension assembly consists of a mounting block, suspension beam, flexure and slider. The Hutchinson Type 870 suspension used in this research. The finite element model is created with the undeformed state of suspension. After loaded onto the hard disk, the suspension undergoes a preloading force about $5.5269 \mathrm{~N}$, and the angle between the suspension and the base line of the mounting block reduces from 10 to about 2 degrees. This loading process can be performed by analysis procedures of $\mathrm{ABAQUS}^{1}$, where the user simply divides the loading histories into steps and comes up with the deformed state of pre-stress for 0-7803-3590-2/96\$5.00 ๑ 1996 IEEE. subsequent analyses. The air bearing that separates the slider from the media during the operation is approximated by four linear springs supporting at four corners of the slider.

Finite Element Model. The finite elemen$\mathrm{t}$ model, as shown in Fig. 1, for the suspension assembly includes initially a grand total of $338 \mathrm{el}-$ ements, 439 nodes, with 2551 degrees of freedom. Three shape parameters are selected as design variables by sensitivity analysis: $H_{B A S E}, H_{L}$ and $R_{H}$. The equations of motion of the suspension assembly for the finite element model of order $N$ have the form

$$
M(\mathbf{x}) \ddot{\mathbf{q}}+C(\mathbf{x}) \dot{\mathbf{q}}+K(\mathbf{x}) \mathbf{q}=B \mathbf{u}
$$

where $\mathbf{x}$ is a vector of design variables, $M(\mathbf{x})$ is a positive definite symmetric mass matrix, $K(\mathbf{x})$ is the nonnegative symmetric stiffness matrix, and $\mathbf{q}$ is the generalized nodal coordinates. The first 4 natural frequencies of the original suspension in the unloaded and loaded positions are compared in Table 1.

\section{Integrated Structure and Control optimization}

The multiobjective optimization problem can be stated as follows:

Minimize the cost functionals

$$
f_{1}=\frac{1}{\omega_{1}}, \quad f_{2}=\frac{1}{\omega_{2}}, \quad f_{3}=\frac{1}{\omega_{3}-\omega_{2}}
$$

subject to the minimum and maximum values of design variables.

It is efficient and practical for the designer to truncate those modal coordinates that correspond to high-frequency modes. In the following optimal control formulation, selected are 10 modal coordinates that describe the dominant dynamic behavior of the suspension assembly. In order to design a 
linear quadratic regulator a performance index $(P I)$ can be defined as

$$
f_{4}=\frac{1}{2} \int_{0}^{\infty}\left(\overline{\mathbf{q}}^{T} \mathbf{Q} \overline{\mathbf{q}}+\mathbf{u}^{T} \mathbf{R} \mathbf{u}\right) d t
$$

where $\overline{\mathbf{q}}=\left[\mathbf{q}^{T} \dot{\mathbf{q}}^{T}\right]^{T}$, and $\mathbf{Q}$ and $\mathbf{R}$ are weighting matrices.

Table 1 Natural frequencies of suspension assembly

\begin{tabular}{|c|c|l|l|l|}
\hline & \multicolumn{2}{|c|}{ Unloaded } & \multicolumn{2}{c|}{ Loaded } \\
\hline Mode & Freq. & Type & Freq. & Type \\
1 & 2282 & bending & 2241 & bending \\
2 & 2606 & torsional & 2770 & torsional \\
3 & 5732 & torsional & 6813 & bending \\
4 & 6668 & bending & 6974 & torsional \\
\hline
\end{tabular}

Optimization Results

The optimization of the suspension assembly is investigated with two techniques: goal programming and compromise programming [1] coded in C language. The ABAQUS is called internally by MOST whenever the structural analysis for eigenvalues and eigenvectors are requested [2]. The weighting matrix $\mathbf{Q}$ in (3) is chosen as $10^{14} \mathbf{I}$ and $\mathbf{R}=10$ by experiences.

Table 2 Active design results

\begin{tabular}{|c|c|c|}
\hline & $\beta=1$ & $\gamma=1$ \\
\hline$H_{B A S E}(m m)$ & 1.370 & 1.370 \\
\hline$H_{L}$ & 6.000 & 6.000 \\
\hline$R_{H}$ & 0.463 & 0.393 \\
\hline \hline$\omega_{1}(\overline{H z})$ & 3052.1 & 2911.4 \\
\hline$\omega_{2}$ & 3483.5 & 3166.1 \\
\hline$\omega_{3}-\omega_{2}$ & 4174.2 & 4435.5 \\
\hline$C O S T$ & 2.4167 & 2.3312 \\
\hline
\end{tabular}

Active design with both vertical and lateral control efforts. It is adequate to implement the control forces independently in both the $y$ and $z$ directions on the yz-plane. Figure 2 shows the Bode plots of the active design obtained by the compromise programming with independent control forces in the $y$ and $z$-directions, and all the bending and torsional modes are perfectly suppressed. Table 2 shows the corresponding values of design variables, natural frequencies and costs. The optimal shape in the finite element mesh for the case of $\gamma=1$ is presented in Fig. 3.

\section{Summary and Conclusions}

The multiobjective optimization design of the suspension assembly of hard disk drives has been $p$ resented. The final solution may not be unique, but depends on additional engineering experience, manufacturing requirements, and so on.

\section{Acknowledgment}

This research was supported by National Science Council under Contract No. NSC83-0422-E-002-005.

\section{References}

[1] Tseng, C. H., Liao, W. C. and Yang, T. C., MOST User's Manual Version 1.1, Technical Report No. AODL-93-01, National ChiauTung University, Taiwan, R.O.C., 1993.

[2] Yang, Y. P. and Tseng, C. H., "Multiobjective Optimization of Complex Structures Integrated with Finite Element Software on Workstations," Journal of the Chinese Society of Mechanical Engineers, Vol. 16, No. 2, 1995, pp. 167-179.

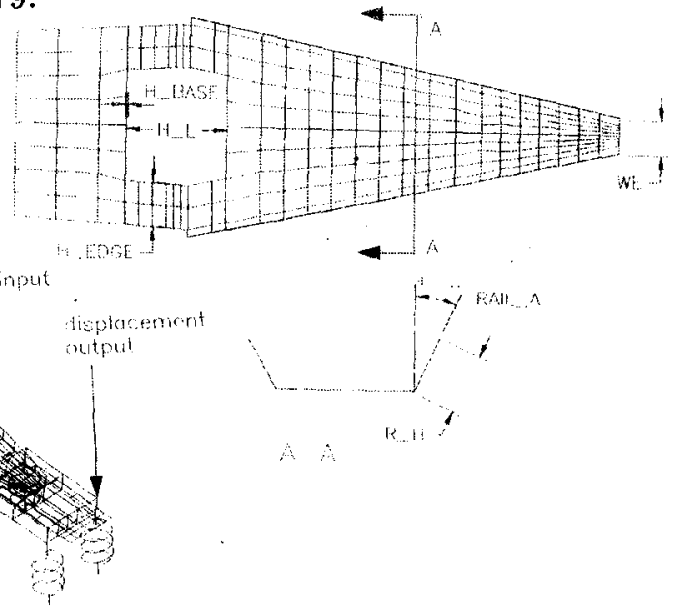

Fig. 1 Finite element model of suspension assembly

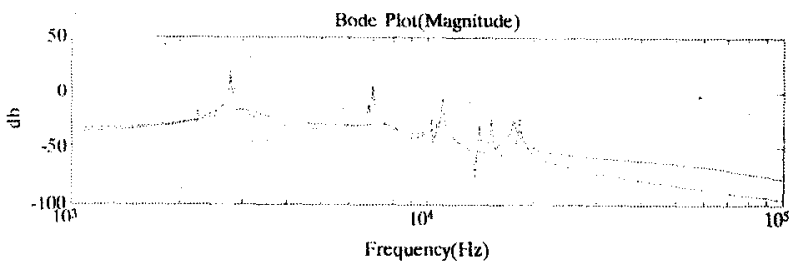

Fig. 2 Closed-loop frequency response of suspension by active design

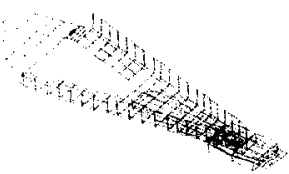

Fig. 3 Optimal shape of active design 